\title{
Dificuldades relatadas por estudantes de odontologia diante de procedimentos relacionados à periodontia
}

\author{
Maurício Meirelles Nagle*, Ana Cláudia Gabrielli Piveta**, Weber Adad Ricci*, \\ Andréia Affonso Barretto Montandon**
}
* Prof. Adjunto Doutor do Departamento de Odontologia Social, Disciplina de Clínica Integrada da Faculdade de Odontologia de Araraquara da Universidade Estadual Paulista
** Doutora em Reabilitação Oral e estagiária do Departamento de Odontologia Social, Disciplina de Clínica Integrada da Faculdade de Odontologia de Araraquara da Universidade Estadual Paulista

\section{RESUMO}

Objetivo: O presente trabalho teve por objetivo avaliar as principais dificuldades relatadas por estudantes do último ano do curso de Odontologia na execução de procedimentos periodontais básicos e cirúrgicos. Métodos: Um questionário proposto com 24 questões, sendo 14 referentes a procedimentos periodontais básicos e 10 a procedimentos cirúrgicos foi aplicado em 45 alunos de graduação com trabalhos envolvendo tais procedimentos durante as atividades práticas da Disciplina de Clínica Integrada, disciplina obrigatória da grade curricular do curso, ministrada aos alunos do último ano. Os alunos foram questionados quanto à percepção individual de dificuldades quanto a execução dos diversos passos referentes aos tratamentos avaliados. O grau de dificuldade individual foi determinado segundo a escala Likert em 0 = nenhuma, 1 = leve, 2 = moderada, $3=$ alta e $4=$ muito alta. Aplicou-se a análise exploratória dos dados obtidos e adicionalmente, o grau de dificuldade foi dicotomizado em nenhuma a leve (escores 0 e 1) e moderada a muito alta (escores 2 a 4). Resultados: Com relação ao tratamento periodontal básico, a sondagem do nível ósseo foi o procedimento com maior quantidade de alunos em dificuldade $(57,5 \%)$. Para as dificuldades relatadas por estudantes relacionadas às etapas de uma cirurgia periodontal apenas os procedimentos relacionados a anestesia e remoção de sutura não apresentaram relatos de dúvidas. Conclusão: O procedimento cirúrgico periodontal foi o que envolveu maior prevalên- cia de dúvidas relatadas de forma moderada a muito alta, sendo a indicação da técnica cirúrgica adequada, a etapa com a maior porcentagem de dúvidas $(66,6 \%)$ entre os graduandos.

\section{DESCRITORES}

Odontologia. Ensino. Periodontia.

$\Delta$ s Diretrizes Curriculares para os Cursos de Odontologia publicadas em 2002 (Resolução CNE/CES 3, 2/2002), , ${ }^{2}$ estabelecem como objetivo fundamental dos cursos de graduação, a formação de um generalista, que compreenda os determinantes sociais, culturais, comportamentais, psicológicos, ecológicos, éticos e legais; nos níveis individual e coletivo do processo saúde-doença, que possua uma sólida formação técnico-científica e ético-humanística, que sejam críticos e reflexivos, sendo capazes de trabalhar em equipes multiprofissionais e de dar respostas às necessidades sociais no âmbito da profissão. ${ }^{3}$

A integração dos conteúdos essenciais visa o desenvolvimento de habilidades e competências que permitirá ao futuro profissional, agir com resolutividade e ética na solução de problemas em nível populacional e individual. Assim, a formação do cirurgião dentista tem por objetivo atuar em todos os níveis de atenção à saúde, sendo um dos maiores cuidados do processo educativo, a construção do conhecimento por parte do aluno, a partir de um professor que atue como facilitador e mediador do processo ensino- 
aprendizagem, entendendo seu papel na transformação social. ${ }^{2}$

Assim, no processo ensino-aprendizagem em Odontologia é importante a elaboração de atividades que permitam ao aluno o desenvolvimento progressivo da habilidade, conhecimento técnico e compreensão dos conteúdos curriculares, e segundo Dias Sobrinho ${ }^{1}$, a aprendizagem é uma das mais importantes dimensões da educação.

Sendo assim, os processos avaliativos devem compreender vários aspectos da complexidade desse fenômeno, pois a aprendizagem não se reduz a seus elementos visíveis, isto é, aquilo que pode ser demonstrado, e tampouco se limita aos objetivos práticos mais imediatos.

Indicadores subjetivos têm sido utilizados de forma crescente na literatura com a finalidade de melhor conhecimento de parâmetros como saúde e qualidade de vida, ${ }^{12}$ sendo escassos os estudos que buscam informações sobre o aprendizado relatado por graduandos de odontologia quanto a procedimentos específicos. ${ }^{11,7}$

O estudo da periodontia é considerado exigir uma formação constante e intensa, não sendo suficiente para esta formação, o ensino presente no currículo de graduação. ${ }^{6}$

Em 2008, Togashi et al. ${ }^{13}$ utilizaram mandíbula de porco com a finalidade de facilitar a vivência dos alunos de técnica cirúrgica durante o treinamento laboratorial de periodontia, evidenciando a importância deste treinamento, bem como suas dificuldades.

Medeiros $^{7}$ e Saini et al. ${ }^{10}$ ressaltaram a importância em identificar as causas de erros cometidos por estudantes de odontologia durante o aprendizado, e se estes ocorrem em função da falta de habilidade ou negligência. Contudo, o objetivo fundamental deste estudo foi identificar dificuldades relatadas diante das fases do tratamento que despertam maiores dúvidas, inseguranças e portanto, menor autonomia por parte do estudante do último ano de graduação em odontologia, residindo neste as limitações do presente estudo.

Assim, considerando a alta prevalência das doenças periodontais na população adulta, a importância de sua correta abordagem ${ }^{10,8,5}$ e conseqüente necessidade de conhecimento por parte do clínico geral dos padrões de diagnóstico, intervenção precoce, tratamento e abordagem cirúrgica com finalidade restauradora, este estudo teve por objetivo fundamental conhecer o padrão de dificuldades relatadas por graduandos do último ano do curso de odontologia fren- te à necessidade de execução de procedimentos periodontais.

\section{MATERIAIS E MÉTODOS}

Um questionário estruturado contendo 24 questões que abordaram passos relevantes do tratamento periodontal básico e cirúrgico, após sua realização, foi aplicado a 45 graduandos do último ano do curso de Odontologia. Estes corresponderam a uma amostra de conveniência correspondente a $60 \%$ dos 75 alunos matriculados na Disciplina de Clínica Integrada, ministrada como conteúdo obrigatório aos alunos do último ano.

O questionário incluiu questões referentes as várias etapas dos tratamento periodontal básico (14 questões) e cirúrgico (10 questões), e a percepção do grau de dificuldade foi classificado segundo a escala Likert em 0 = nenhuma, $1=$ leve, $2=$ moderada, $3=$ alta e $4=$ muito alta para os procedimentos envolvidos no atendimento prestado aos pacientes.

Aplicou-se a análise exploratória dos dados obtidos e adicionalmente, o grau de dificuldade foi dicotomizado em nenhuma a leve (escores 0 e 1 ) e moderado a muito alto (escores 2 a 4), sendo realizado a análise descritiva dos resultados encontrados.

Anteriormente a aplicação dos questionários, um estudo piloto buscou conhecer a reprodutibilidade intra examinadores do questionário proposto a 10 estudantes que o preencheram imediatamente após a realização do procedimento e uma semana após, utilizando-se a estatística kappa para verificação da reprodutibilidade do instrumento.

\section{RESULTADOS}

Após a realização do teste piloto, o instrumento mostrou ter boa reprodutibilidade e validade (kap$\mathrm{pa}=0,84)$.

Baseando-se nos objetivos deste estudo e metodologia empregada, os resultados obtidos estão representados nas Tabelas 1 e 2, e Gráficos 1 e 2.

Com relação ao tratamento periodontal básico, a sondagem do nível ósseo foi o procedimento com maior quantidade de alunos em dificuldade $57,5 \%$, seguido pela marcação do nível ósseo na ficha clínica com 55\%, RPD (Raspagem e polimento dental) no SSE (Sextante Superior Esquerdo) com 52,5\%, e RPD no SSD (Sextante Superior Direito) com 32,5\%. Esses resultados estão dispostos no Gráfico 1.

O Gráfico 2 e a Tabela 2 apresentam as dificuldades relatadas por estudantes relacionadas às etapas de uma cirurgia periodontal. Nesta seqüência, apenas 


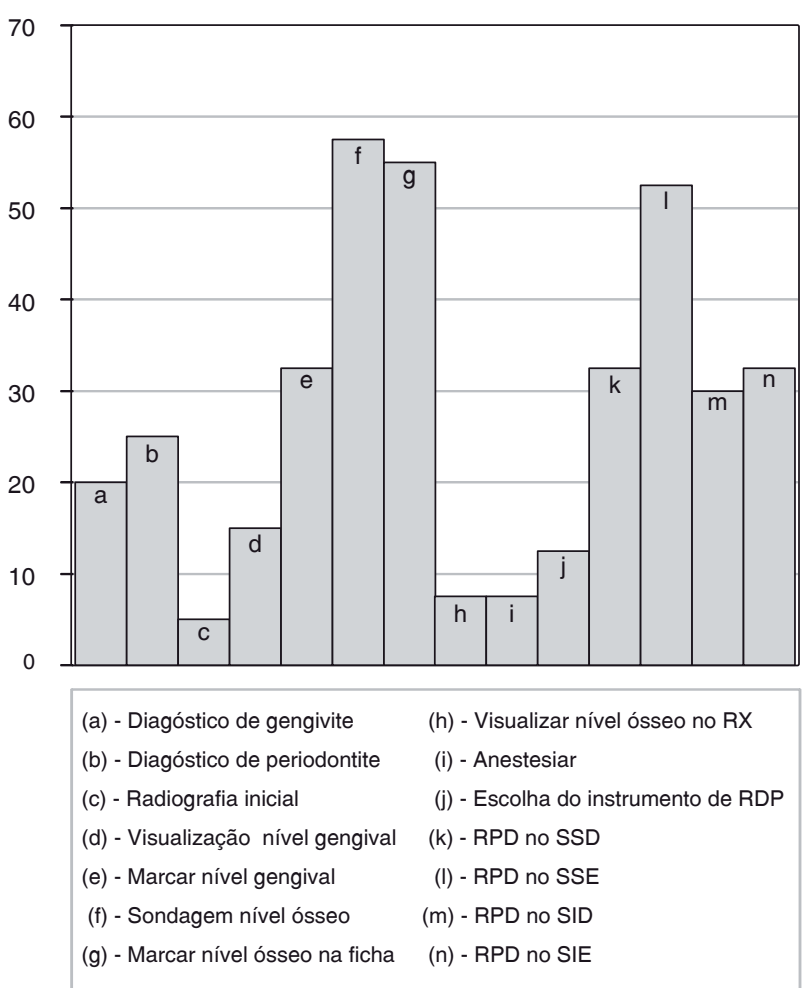

Gráfico 1 - Nível de dificuldade relatado por estudantes de Odontologia durante os procedimentos básicos de periodontia.

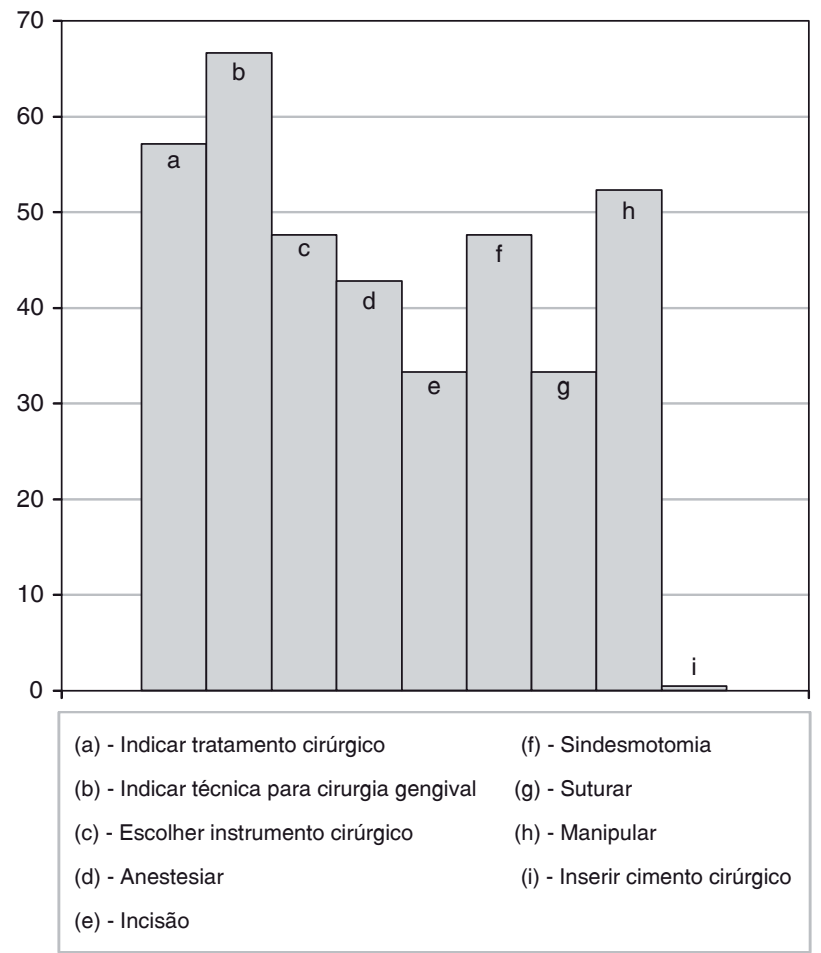

Gráfico 2 - Dificuldade relatada por estudantes de odontologia para os procedimentos cirúrgicos periodontais.
TABELA 1 - Nível de dificuldade dos alunos durante os procedimentos de periodontia.

\begin{tabular}{|c|c|c|c|c|}
\hline \multirow{2}{*}{$\begin{array}{c}\text { Dificuldade } \\
\text { Diagnóstico de gengivite }\end{array}$} & \multicolumn{2}{|c|}{$\begin{array}{l}\text { Nenhuma - } \\
\text { Leve ( } \% \text { ) }\end{array}$} & \multicolumn{2}{|c|}{$\begin{array}{c}\text { Moderada - } \\
\text { Máxima (n \%) }\end{array}$} \\
\hline & 32 & 80 & 8 & 20 \\
\hline Diagnóstico de periodontite & 30 & 75 & 10 & 25 \\
\hline Radiografia Ir & 38 & 95 & 2 & 5 \\
\hline Visualização - nível gengival & 34 & 85 & 6 & 15 \\
\hline Marcar nível gengival & 27 & 67,5 & 13 & 32,5 \\
\hline Sondagem - nível ósseo & 17 & 42,5 & 23 & 57,5 \\
\hline Marcar nível ósse & 18 & 45 & 22 & 55 \\
\hline Visualizar nível ósseo no RX & 37 & 92,5 & 3 & 7,5 \\
\hline Anestesiar & 37 & 92,5 & 3 & 7,5 \\
\hline Escolha do instrumento RPD & 35 & 87,5 & 5 & 12,5 \\
\hline RPD no SSD* & 27 & 67,5 & 13 & 32,5 \\
\hline RPD no SSE & 19 & 47,5 & 21 & 52,5 \\
\hline RPD no SID ${ }^{* \star *}$ & 28 & 70 & 12 & 30 \\
\hline RPD no SIE ${ }^{\star \star \star \star}$ & 27 & 67,5 & 13 & 32,5 \\
\hline
\end{tabular}

${ }^{*}$ raspagem e polimento dental no sextante superior direito, ** raspagem e polimento dental no sextante superior esquerdo, ${ }^{* * *}$ raspagem e polimento dental no sextante inferior direito, ${ }^{* \star \star \star}$ raspagem e polimento dental no sextante inferior esquerdo.

TABELA 2 - Nível de dificuldade dos alunos durante os procedimentos cirúrgicos de periodontia.

\begin{tabular}{|l|c|r|r|r||}
\hline \multicolumn{1}{|c|}{ Dificuldade } & $\begin{array}{l}\text { Nenhuma - } \\
\text { Leve (n \%) }\end{array}$ & \multicolumn{2}{|c|}{$\begin{array}{c}\text { Moderada - } \\
\text { Máxima (n \%) }\end{array}$} \\
\hline $\begin{array}{l}\text { Indicar tratamento cirúrgico } \\
\text { adequado }\end{array}$ & 9 & 42,8 & 12 & 57,1 \\
\hline $\begin{array}{l}\text { Indicar técnica para cirurgia } \\
\text { gengival }\end{array}$ & 7 & 33,3 & 14 & 66,6 \\
\hline $\begin{array}{l}\text { Escolher instrumento } \\
\text { cirúrgico }\end{array}$ & 11 & 52,3 & 10 & 47,6 \\
\hline $\begin{array}{l}\text { Anestesiar } \\
\text { Incisão }\end{array}$ & 21 & 100 & - & - \\
\hline $\begin{array}{l}\text { Sindesmotomia } \\
\text { Suturar }\end{array}$ & 12 & 57,1 & 9 & 42,8 \\
\hline Manipular cimento & 11 & 66,6 & 7 & 33,3 \\
\hline Inserir cimento cirúrgico & 14 & 66,6 & 7 & 33,3 \\
\hline Remoção da sutura & 10 & 47,6 & 11 & 52,3 \\
\hline
\end{tabular}


os procedimentos relacionados a anestesia e remoção de sutura não apresentaram relatos de dúvidas, sendo a indicação da técnica para cirurgia gengival adequada com $66,6 \%$, indicação do tratamento cirúrgico adequado com $57,1 \%$, colocação do cimento na ferida cirúrgica $(52,3 \%)$ escolha do instrumento cirúrgico e sutura $(47,6 \%)$, descolamento do tecido e manipulação do cimento $(33,3 \%)$.

\section{DIscussão}

As Diretrizes Curriculares Nacionais para os Cursos de Odontologia consideram que generalistas devem ser formados a partir dos cursos de graduação com perfil direcionado às necessidades sociais da população. ${ }^{2}$

Assim, a compreensão dos conteúdos curriculares torna-se fundamental para sua formação, ${ }^{1}$ embora especificamente referindo-se a periodontia, esta seja considerada uma área de extenso conhecimento sem possibilidade de ser totalmente ministrado durante o curso de graduação. ${ }^{6,13}$

Quando os docentes possibilitam aos alunos o confronto entre o aprendizado teórico e as situações reais, a compreensão se torna mais significativa e a experiência enriquecedora. Assim, o ensino integrado tem por objetivo agrupar as atividades práticas embasadas no conteúdo teórico, estimulando a compreensão e a vivência da profissão, colocando diante do estudante, além dos desafios específicos de cada área do conhecimento, aqueles relativos ao planejamento integrado, em que a responsabilidade de uma fase está diretamente relacionada ao sucesso da outra.

O atendimento do paciente com quadro clínico de doença periodontal pode se tornar complexo diante da falta de adequada abordagem ${ }^{10,8,5}$ e em função da importância dos tecidos periodontais na promoção de saúde. Assim, o resultado do tratamento periodontal proposto não depende apenas do conhecimento sobre a importância dos fatores relacionados ou da remoção do biofilme dental ${ }^{9,4}$ e habilidade do profissional, mas também da preocupação do profissional frente ao desafio de educar o paciente para o controle da placa bacteriana.

Quanto às dificuldades relatadas diante de necessidade de diagnóstico de gengivite e periodontite, assim como tomadas radiográficas iniciais, observouse que as dificuldades de diagnóstico da periodontite (25\%) foram maiores em relação ao diagnóstico da gengivite (20\%), segundo o relato dos estudantes.

Adicionalmente, entre os que relataram dificuldades para os procedimentos de raspagem e alisamento radicular, $52,5 \%$ das maiores dificuldades ocorreram no quadrante superior esquerdo, sendo estas, menores nos sextantes superiores e inferiores direito, provavelmente em função do posicionamento e visibilidade obtida pelo estudante.

A cirurgia periodontal é uma forma apropriada de terapia para obtenção de visibilidade, acesso ao preparo radicular e remoção de irritantes locais adjacentes às bolsas profundas com envolvimento de furca, podendo ainda obter um meio de controle da doença onde métodos não cirúrgicos foram ineficientes. $^{9}$

Segundo Togashi et al. ${ }^{13}$, o treinamento das técnicas de manipulação dos tecidos ósseo e mole, cirurgias regenerativas, pré-protéticas, amputação e hemissecção pode ser empregado em nível mais avançado do aprendizado, como nos cursos de pós-graduação, por apresentarem maior grau de complexidade.

Contudo, o planejamento das cirurgias periodontais deve ser realizado de forma a possibilitar que os estudantes elaborem na prática, o que aprendem na teoria, vivenciando situações complexas e os mais variados tipos de situações clínicas. Na disciplina de Clínica Integrada, entre os estudantes avaliados, foram realizadas 21 cirurgias periodontais a retalho decorrentes do planejamento dos tratamentos clínicos integrados.

Os resultados encontrados no presente estudo confirmam as dificuldades apresentadas pelos estudantes quando se tratou, principalmente, de procedimentos cirúrgicos. Considerando-se a correta indicação da técnica cirúrgica, $66,6 \%$ dos estudantes relataram dificuldades classificadas como moderadas a máximas, sendo de $57,1 \%$ para a simples indicação do tratamento cirúrgico e $47,6 \%$ para a escolha do instrumento cirúrgico ou execução da sutura, resultados esperados para procedimentos complexos executados por estudantes de graduação.

Portanto, a atividade clínica não deve ser vista apenas como uma maneira de desenvolver habilidade e competência técnica nos domínios de aspectos biológicos envolvidos na prática clínica, mas, essencialmente, o aluno deve ser preparado para discernir quanto à dimensão ética da sua atuação, ${ }^{13}$ devendo ter consciência de suas dificuldades, de seus limites e necessidade de contínuo aprendizado.

\section{CONCLUSÃO}

De acordo com os resultados obtidos e a metodologia empregada, pode-se concluir que os procedimentos peridontais que levam a um maior relato de dificuldades consideradas medianas ou máximas por estudantes de odontologia são os procedimentos de 
diagnóstico da periodontite por sondagem e indicação da técnica cirúrgica.

\section{ABSTRACT}

Problems reporterd by dental students about procedures related to periodontics

Objective: This study aimed at evaluating the difficulties reported by last-year dentistry course students regarding basic and surgical periodontal procedures. Methods: A proposed questionnaire with 24 questions, 14 related to basic periodontal procedures and 10 to surgical procedures, was applied to 45 undergraduate students with jobs involving these procedures, during the practical activities of the Integrated Clinic Discipline, a compulsory subject in the curriculum of the course, taught to last-year students. Students were asked about their own perceptions of the difficulties in implementing the various steps related to the treatments. The degree of individual difficulty was determined according to the Likert scale: $0=$ none, $1=$ mild, $2=$ moderate, $3=$ high, $4=$ very high. Exploratory analysis of data was applied, and the difficulty level was also dichotomized into none to mild (scores 0 and 1) and moderate to very high (scores 2-4). Results: Regarding the basic periodontal treatment, bone level probing was the procedure with the highest number of student difficulties $(57.5 \%)$. As for the difficulties reported by students regarding the stages of periodontal surgery, only the procedures related to anesthesia and suture removal had no report of doubts. Conclusion: Periodontal surgery was the procedure involving the most prevalent questions reported as moderate to very high, and the indication for the most suitable surgical technique was the stage with the highest percentage of questions (66.6\%) among the undergraduates.

\section{DESCRIPTORS}

Dentistry. Education. Periodontics.

\section{REFERÊNCIAS}

1. Dias Sobrinho J. Avaliação - políticas e reformas da educação superior. São Paulo: Cortez; 2003.

2. Diretrizes do Curso de Odontologia, Revista da ABENO. 2001; 2(1):31-34.

3. Fernandes Neto AJ A evolução dos cursos de Odontologia no Brasil. Revista da ABENO. 2002; 2(1):55-56.

4. Gottehrer NR, Martin JL. A future for periodontal therapy. Dent Today. 2010; 29(2):154, 156-7

5. Liang S, Hosur KB, Domon H, Hajishengallis G. Periodontal inflammation and bone loss in aged mice. J Periodontal Res. 2010; 45(4):574-8.

6. Lotufo R. Roberto Lotufo: entrevista. Dental Press Periodontia Implantol; 2007; 1(4): 15-24.

7. Medeiros JMF., Habitante SM., Zöllner NA., Carvalho PL, Pinto CA, Lage-Marques JL. Análise das dificuldades dos alunos de graduação durante as manobras de odontometria. Revista da ABENO 2001; 7(1):38-46.

8. Oliveira AN. Estudos sobre a influência de diferentes níveis de conhecimento sobre saúde bucal na distribuição da placa e medidas de higiene bucal. Rev. Dental. Press Periodontia Implantol. 2007; 1(1):46-59.

9. Rosenberg, M. M.; Kay, H. B.; Keough, B. E.; Holt, R. L. Tratamento periodontal e protético para casos avançados . 2. ed. São Paulo: Quintessence, 1992

10. Saini R, Marawar PP, Shete S, Saini S. Periodontitis, a true infection. J Glob Infect Dis. 2009; 1(2):149-50.

11 Simi Junior J, Medeiros JMF, Risso VA, Albetman CS. Avaliação das dificuldades clínicas identificadas por acadêmicos do curso de graduação em relação as diversas etapas do tratamento endodontico. Odontologia. USF. 1998; 16(1):11-18.

12 Slade GD. Derivation a validation of a short-form oral health impact profile. Community Dentistry Oral Epidemiology 1997; 25:284-290

13 Togashi AY, Corraini P, Francisco Emilio Pustiglioni FE, Alves de Lima LAP, Georgetti MAP. Prática laboratorial de cirurgia periodontal: otimizando o ensino-aprendizagem Além de desenvolver habilidade e competência técnica, a atividade préclínica prepara o aluno para discernir quanto à dimensão ética de sua atuação. Revista da ABENO, 2008; 8(1:)5-8. 